\title{
Optimizing energy production systems under uncertainty
}

Arne B. Huseby

University of Oslo, Norway

Electricity infrastructure has become a critical element of modern industrial society. In order to model and analyse this infrastructure, identify weaknesses, and optimize performance, one needs to take into account its distributed nature. Rather than modelling a single system, energy production and distribution systems consists of many more or less autonomous subsystems working together and trading with each other. Analytical models could perhaps be used to describe a single subsystem. However the complexity related to the interactions between the subsystems soon becomes unmanageable. Even establishing a simulation model for such phenomenons is a non-trivial task, especially if the model is required to be easily scaleable. In this paper we consider the problem of optimizing a simplified energy system with respect to supply stability. This is done using both deterministic methods and Monte Carlo methods. The system is broken into smaller units. These units may trade energy between them in order to maintain a stable supply covering the demand. An important element in the model is the ability to store energy within the unit. For some units, e.g., hydroelectric power plants, the energy can be easily stored in the form of a water reservoir. For other units, like wind power plants, storing energy is usually not feasible. By using an object oriented software framework, we can compare different production units, and study how these can interact in order to facilitate a stable total production.

\section{INTRODUCTION}

Electricity infrastructure has become a critical element of modern industrial society. In order to model and analyse this infrastructure, identify weaknesses, and optimize performance, one needs to take into account its distributed nature. Rather than modelling a single system, energy production and distribution systems consists of many more or less autonomous subsystems working together and trading with each other. Explicit analytical models can often be used to describe a single subsystem. However the complexity related to the interactions between the subsystems soon becomes unmanageable. Even establishing a simulation model for such phenomenons is a non-trivial task, especially if the model is required to be easily scaleable. Various types of modelling tools have been introduced in order to simulate such complex systems. See (Axelrod 1997) and (Kremers 2013).

In this paper we consider a simplified system consisting of a set of energy production units. For a related problem see (Palensky and Dietrich 2011). For some units, e.g., hydroelectric power plants, the energy can be easily stored in the form of a water reservoir. For other units, like wind power plants, storing energy is usually not feasible. Our main goal is to study how the different producers and consumers can interact in order to facilitate a stable total production. We start out by considering a simplified version of the problem where an optimal production strategy can be derived analytically. Using this as a starting point we proceed to a more general setting, and study the robustness of the derived strategy. It turns out that the simple solution behaves very well in a wide range of cases.

\section{BASIC CONCEPTS AND RESULTS}

The approach used in this section is similar to the methodology introduced in (Huseby and Haavardsson 2009) in the context of oil production. See also (Huseby and Haavardsson 2010). We consider the energy production from a set of $n$ producers serving a common set of consumers. The energy demand from the consumers at time $t \geq 0$ is constant $K(t)>0$, expressed in some suitable unit, e.g., TWh.

Each producer has a reservoir where energy can be stored in some alternative form, e.g., as potential energy of water in a reservoir. Let $\boldsymbol{R}(t)=\left(R_{1}(t), \ldots, R_{n}(t)\right)$ denote the vector of stored energy for the $n$ producers, and let $\boldsymbol{f}(t)=\left(f_{1}(t), \ldots, f_{n}(t)\right)$ be the corresponding vector of potential production rate functions, which we refer to as the PPR-functions. We assume that the potential production rate from a producer can be expressed as a function of the stored energy at the same reservoir. More specifically, we assume that for $i=1, \ldots n$ the $i$ th PPR-function can be written as:

$$
f_{i}(t)=f_{i}\left(R_{i}(t)\right)=\min \left(a_{i} R_{i}(t), b_{i}\right), \quad t \geq 0 .
$$

Here the factors $a_{1}, \ldots, a_{n}$ are numbers between 0 and 1 representing restrictions on the productions imposed in order to prevent the reservoirs from being emptied too quickly. The constants $b_{1}, \ldots, b_{n}$, on the other hand represent limitations in the production facilities.

Under normal operational conditions the amount of energy stored in a reservoir will of course vary both up and down. Production of energy to the consumers will reduce the amount of energy in the reservoir, while the inflow of energy, e.g., through precipitation, will increase the amount 
of energy. While the production can be controlled by a production manager, the inflow of energy is typically uncertain. For longer or shorter periods the inflow may even be zero. A sensible production strategy should take this uncertainty into acount and minimize the risk of an energy shortage.

In general the actual production from the $n$ producers depends both on the PPR-functions and how the production is distributed between the producers. Whenever the total availabel production capacity is greater than the demand, the total production rate from the producers will be less than the sum of their potential rates.

The actual production rates from the reservoirs, after the production is adjusted to satsify the demand $K(t)$, are represented by the vector $\boldsymbol{r}(t)=\left(r_{1}(t), \ldots, r_{n}(t)\right)$.

A production strategy is defined by a vector valued function $\boldsymbol{w}=\boldsymbol{w}(t)=\left(w_{1}(t), \ldots, w_{n}(t)\right)$, defined for all $t \geq 0$, where $w_{i}(t)$ represents the adjustment factor, i.e., the fraction of the potential production rate of the $i$ th producer that is actually produced at time $t, i=1, \ldots, n$. Thus, we have:

$$
0 \leq w_{i}(t) \leq 1, \quad i=1, \ldots, n, \quad t \geq 0 .
$$

The actual production rates can then be expressed as:

$$
r_{i}(t)=w_{i}(t) f_{i}\left(R_{i}(t)\right), \quad i=1, \ldots, n .
$$

The adjustment factors must be chosen such that the constraint (2) is satisfied, and such that:

$$
\sum_{i=1}^{n} w_{i}(t) f_{i}\left(R_{i}(t)\right) \leq K(t) .
$$

Let $\mathcal{W}$ denote the class of production strategies that satisfy these constraints. We refer to production strategies $\boldsymbol{w} \in \mathcal{W}$ as valid production strategies.

Obviuosly, the production should be adjusted so that the demand is satisfied whenever possible. An admissible production strategy is defined as a production strategy $\boldsymbol{w} \in \mathcal{W}$ where the total production rate from the $n$ producers satisfies the following constraint for all $t \geq 0$ :

$$
\sum_{i=1}^{n} w_{i}(t) f_{i}\left(R_{i}(t)\right)=\min \left\{K(t), \sum_{i=1}^{n} f_{i}\left(R_{i}(t)\right)\right\} .
$$

Moreover, we let $\mathcal{W}^{\prime} \subseteq \mathcal{W}$ denote the class of admissible strategies.

We observe that by applying an admissible production strategy, the total production rate is kept at the maximum level $K(t)$ as long as this is possible. Moreover, when it is not possible to satisfy the demand, we have $w_{i}(t)=1, i=$ $1, \ldots, n$. When trying to find the best production strategies, only admissible strategies will be considered.

\section{ZERO INFLOW - CONSTANT DEMAND}

In this section we consider a simplified problem where we assume that $K(t)=K$ for all $t \geq 0$. That is, the total energy demand from the consumers is constant. Moreover, we assume that for an indefinite periode, starting at time $t=0$, the energy inflow to the reservoirs is zero. Finally, we assume that the total production capacity at time $t=0$ satisfies:

$$
\sum_{i=1}^{n} f_{i}\left(R_{i}(0)\right)>K \text {. }
$$

Thus, at least in the beginning of the period under consideration, the total production capacity satisfies the demand. In this case we can introduce the concept of the plateau length for a given production strategy $\boldsymbol{w} \in \mathcal{W}^{\prime}$ defined as

$$
T=T(\boldsymbol{w})=\sup \left\{t \geq 0: \sum_{i=1}^{n} f_{i}\left(R_{i}(t)\right) \geq K\right\} .
$$

The objective is to find a production strategy which maximizes $T$.

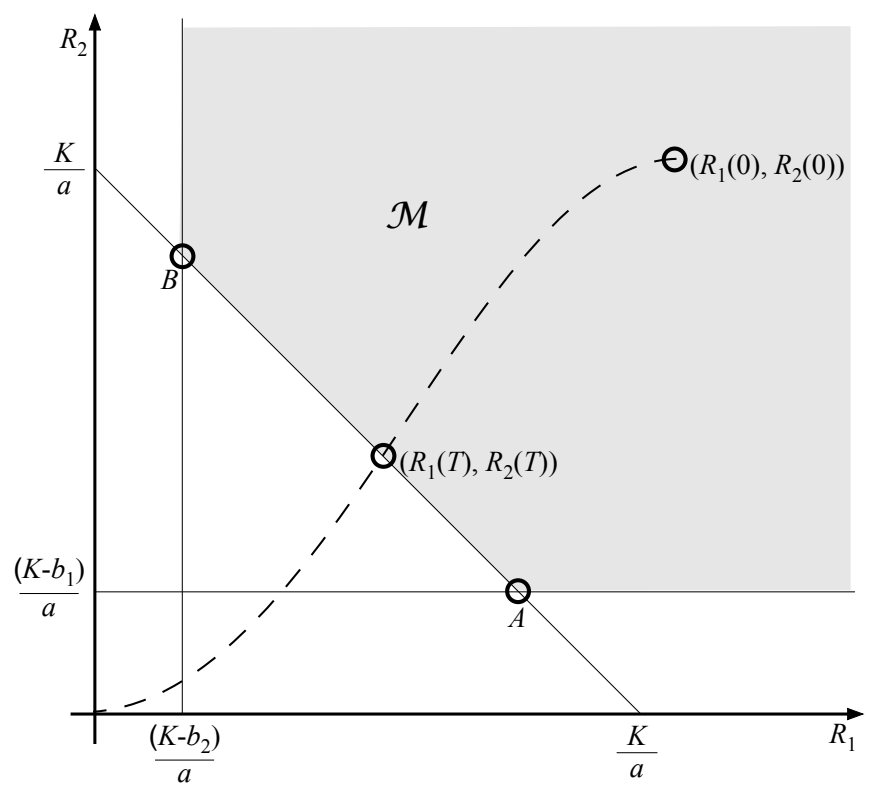

Figure 1: Energy production illustrated as a path.

In order to analyse this problem, we introduce the following set:

$$
\mathcal{M}=\left\{\boldsymbol{R} \in\left(\mathbb{R}^{+}\right)^{n}: \sum_{i=1}^{n} f_{i}\left(R_{i}\right) \geq K\right\} .
$$

Thus, the set $\mathcal{M}$ is the set of reservoir states where the demand can be satisfied by the total potential production rate. The situation is illustrated in Figure 1 in the 2-dimensional case, i.e., where $n=2$. The gray region in the figure is the set $\mathcal{M}$.

By combining (1) and (8) it follows that any point $\boldsymbol{R} \in$ $\mathcal{M}$ satisfies the following equation:

$$
\sum_{i=1}^{n} \min \left(a_{i} R_{i}, b_{i}\right) \geq K
$$

This inequality can be expanded to a set of $\left(2^{n}-1\right)$ nontrivial linear inequalities, each of which defines a halfspace in $\mathbb{R}^{n}$, and the set $\mathcal{M}$ is an $n$-dimensional convex polytope obtained as the intersection of all these halfspaces. 
We illustrate this for the case where $n=2$ and where we have simplified the model so that $a_{1}=a_{2}=a$. In this case the condition (9) can be expanded to the following four inequalities:

$$
\begin{aligned}
a R_{1}+a R_{2} & \geq K, \\
a R_{1}+b_{2} & \geq K, \\
b_{1}+a R_{2} & \geq K, \\
b_{1}+b_{2} & \geq K .
\end{aligned}
$$

Here the last inequality, (13), which do not depend on the states of the reservoirs, is trivially satisfied by the assumption (6). Moreover, the inequalities (10), (11) and (12) can be rewritten as:

$$
\begin{aligned}
R_{1}+R_{2} & \geq \frac{K}{a}, \\
R_{1} & \geq \frac{K-b_{2}}{a}, \\
R_{2} & \geq \frac{K-b_{1}}{a} .
\end{aligned}
$$

In Figure 1 the border of the set $\mathcal{M}$ is composed by three straight lines corresponding to the inequalities (14), (15) and (16). In particular, the condition (14) corresponds to the halfspace on the upper right-hand side of the diagonal line through the points $(K / a, 0)$ and $(0, K / a)$. Moreover, the condition (15) corresponds to the halfspace on the right-hand side of the vertical line through the point $\left(\left(K-b_{2}\right) / a, 0\right)$, while the condition (16) corresponds to the halfspace above the horizontal line through the point $\left(0,\left(K-b_{1}\right) / a\right)$. The set $\mathcal{M}$ is the intersection of all these halfspaces.

At time $t=0$, it follows by (6) that the state of the reservoirs, given by the point $\boldsymbol{R}(0)$ is somewhere in the interior of $\mathcal{M}$. As the energy is produced, the state of the reservoirs moves along a path towards the origin. This path is illustrated in Figure 1 by the dashed line. As long as the state is inside $\mathcal{M}$ it possible to satisfy the energy demand. However, eventually, at the point of time $T$, the path reaches the border of $\mathcal{M}$, and then leaves this set. At time $T$ we know that the total produced energy is equal to $K T$. On the other hand this energy can also be expressed as the sum of reduction in energy stored in the $n$ reservoirs. Thus, we have the following equation:

$$
\sum_{i=1}^{n} R_{i}(0)-\sum_{i=1}^{n} R_{i}(T)=K T .
$$

By (17) it follows that maximizing the time $T$ is equivalent to minimizing the remaining energy at time $T$, i.e., $\sum_{i=1}^{n} R_{i}(T)$. Finding a candidate for the optimal point $\boldsymbol{R}(\bar{T})$ can be found by solving the following linear opti- mization problem:

$$
\begin{aligned}
\text { Minimize: } & \sum_{i=1}^{n} R_{i}, \\
\text { subject to: } & \sum_{i=1}^{n} \min \left(a_{i} R_{i}, b_{i}\right) \geq K \\
\text { and: } & R_{i} \geq 0, \quad i=1, \ldots, n .
\end{aligned}
$$

Note that if $b_{i} \geq K$ for some $i$, all linear inequalities involving $b_{i}$ are trivially satisfied, and can be eliminated form the set of inequalities. In particular, if $b_{i} \geq K$ for $i=1, \ldots, n$, the only non-trivial inequality is:

$$
\sum_{i=1}^{n} a_{i} R_{i} \geq K
$$

In this case it can easily be shown that the optimal strategy is to use a socalled strict priority rule where we allocate as much demand as possible to the producer with the lowest $a_{i}$. Then we move on to the producer with the second lowest $a_{i}$ and allocate as much of the remaining demand to this producer, and so on.

Another special case of interest is the one where $a_{1}=$ $\cdots=a_{n}=a$. As the $a_{i}$ s are chosen according to some perhaps common policy, this case is not as unrealistic as it may seem. In this case one of the inequalities can be written as:

$$
\sum_{i=1}^{n} R_{i} \geq K / a
$$

Since the objective is to minimize $\sum_{i=1}^{n} R_{i}$, it follows that any point in $\mathcal{M}$ such that $\sum_{i=1}^{n} R_{i}=K / a$ is optimal. By (6) it follows that $\mathcal{M}$ must contain at least one such point. In fact there will in general not be a unique optimal solution in this case. Moreover, for this case we get by using (17) that:

$$
\sum_{i=1}^{n} R_{i}(0)-\sum_{i=1}^{n} R_{i}(T)=\sum_{i=1}^{n} R_{i}(0)-K / a=K T .
$$

Thus, the maximal plateau length is given by:

$$
T=K^{-1} \sum_{i=1}^{n} R_{i}(0)-a^{-1}
$$

Note that the expression for $T$ does not depend on the $b_{i}$ s.

Now, it should be emphasized that the solution to the linear optimization problem only results in possible candidates for the optimal solution. Due to all the restrictions on the production it may not be possible to find a path which hits the optimal point $\boldsymbol{R}(T)$ on its way out of the set $\mathcal{M}$. Thus, the best one can do is to manage the production so that the point where the path leaves the set $\mathcal{M}$ is as close as possible to an optimal point.

We illustrate this by considering the example shown in Figure 1. In this case the set of optimal points consists of 
the line segment between the points $A$ and $B$. By (6) it follows that $b_{1}+b_{2}>K$. However, if $b_{1}+b_{2}=K+\epsilon$ for some small number $\epsilon>0$, the points $A$ and $B$ will be very close to each other. Depending on the initial state $\boldsymbol{R}(0)$, it may or may not be possible to reach a point between $A$ and $B$. by:

It is easy to see that the coordinates of $A$ and $B$ are given

$$
\begin{aligned}
& A=\left(\frac{b_{1}}{a}, \max \left(0, \frac{K-b_{1}}{a}\right)\right), \\
& B=\left(\max \left(0, \frac{K-b_{2}}{a}\right), \frac{b_{2}}{a}\right) .
\end{aligned}
$$

In particular, if we assume that $b_{i}<K, i=1,2$, we obtain the following simplified coordinates:

$$
\begin{aligned}
& A=\left(\frac{b_{1}}{a}, \frac{K-b_{1}}{a}\right), \\
& B=\left(\frac{K-b_{2}}{a}, \frac{b_{2}}{a}\right) .
\end{aligned}
$$

We now argue heuristically, and consider a point $C$ in the middle of the line segment between $A$ and $B$. Thus, $C$ has coordinates:

$$
C=\left(\frac{K-b_{2}+b_{1}}{2 a}, \frac{K-b_{1}+b_{2}}{2 a}\right) .
$$

In order to maximize the chance of hitting the line segment between $A$ and $B$, we aim at the point $C$. This done by partitioning the space using the straight line through the origin and the point $C$. The slope of this line, denoted $\rho$, is given by:

$$
\rho=\frac{K-b_{1}+b_{2}}{K-b_{2}+b_{1}} .
$$

At any point of time $t \in[0, T]$, we consider the state of the reservoirs, $\left(R_{1}(t), R_{2}(t)\right)$. If $R_{2}(t) / R_{1}(t)<\rho$, this means that the state is below the line trough $C$. Thus, in order to get closer this line, we need to prioritize Producer 1 . That is, we allocate as much of the energy demand as possible to Producer 1. If on the other hand $R_{2}(t) / R_{1}(t)>\rho$, this means that the state is above the line through $C$. Thus, in order to get closer this line, we need to prioritize Producer 2 . Thus, this case we allocate as much of the energy demand as possible to Producer 2.

We now consider a specific numerical example where we let $K=14, a=0.1, b_{1}=14$ and $b_{2}=10$. We notice that $b_{1}=K$. Thus, as long as $a R_{1}(t) \geq K$, Producer 1 can actually produce enough to satisfy the demand. We then calculate the ratio $\rho$ as:

$$
\rho=\frac{14-14+10}{14-10+14}=\frac{10}{18} \approx 0.556 .
$$

The initial states of the reservoirs are assumed to be $R_{1}(0)=400$ and $R_{2}(0)=350$. Thus, $R_{2}(0) / R_{1}(0)=$ $350 / 400=0.875>\rho$. According to the heuristic strategy, we should start out by giving priority to Producer 2 , at least initially.

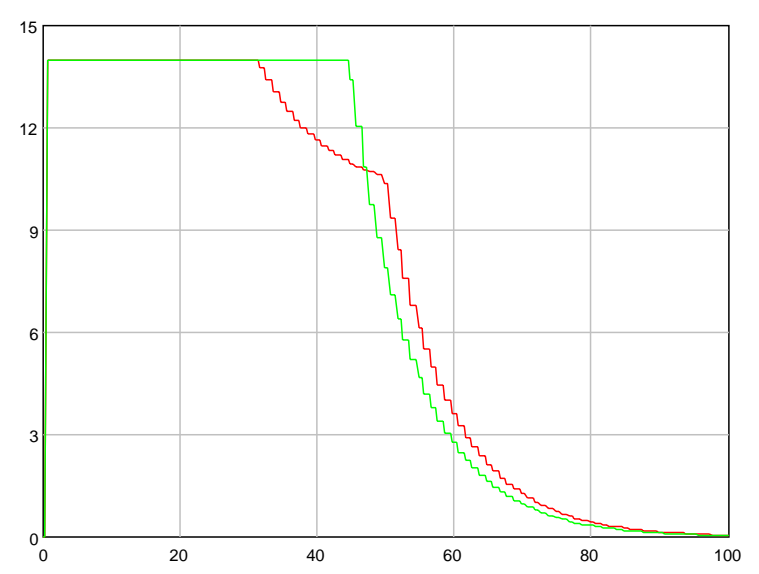

Figure 2: Total production using correct strategy (green curve) and incorrect strategy (red curve).

In Figure 2 we have plotted the total production rate as a function of time given that the heuristic strategy is used. As a comparison we have also plotted the corresponding results given that we use a strategy where the priorities are reversed. Thus, using the reversed strategy we will start out by giving priority to Producer 1 instead. The green curve represents the correct strategy, while the red curve represents the incorrect strategy. We observe that by using the correct strategy, we are able to satisfy the demand for a much longer period. By using the formula for the plateau length (19), we get that:

$$
T=\frac{1}{14}(400+350)-\frac{1}{0.1} \approx 44
$$

It turns out that by using the correct strategy the plateau length is indeed exactly 44 . Thus, we know that this strategy is optimal in this case. If on the other hand the incorrect strategy is used, the plateau length is just 31 .

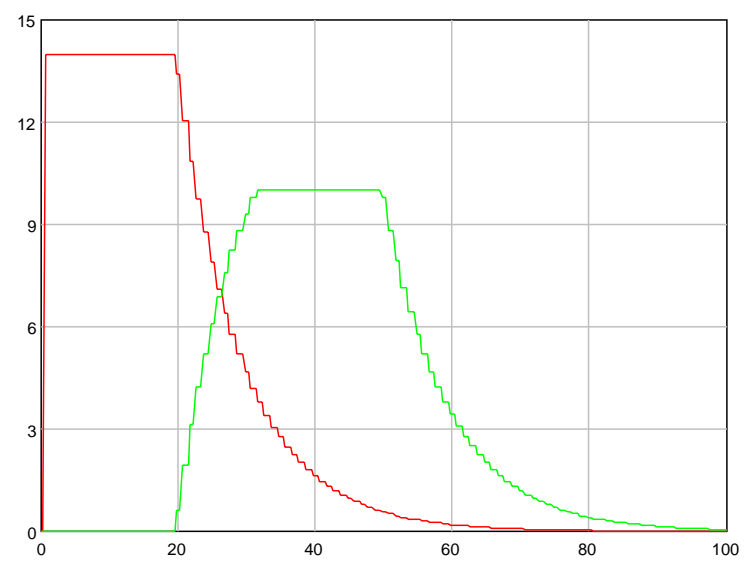

Figure 3: Production from Producer 1 (red curve) and Producer 2 (green curve) using incorrect strategy.

In Figure 3 we have plotted the production from the two producers using the incorrect strategy. The red curve represents Producer 1 while the green curve represents Producer 2. As a consequence of using the incorrect strategy, Producer 1 is given priority in the beginning. Since $b_{1}=K$, 


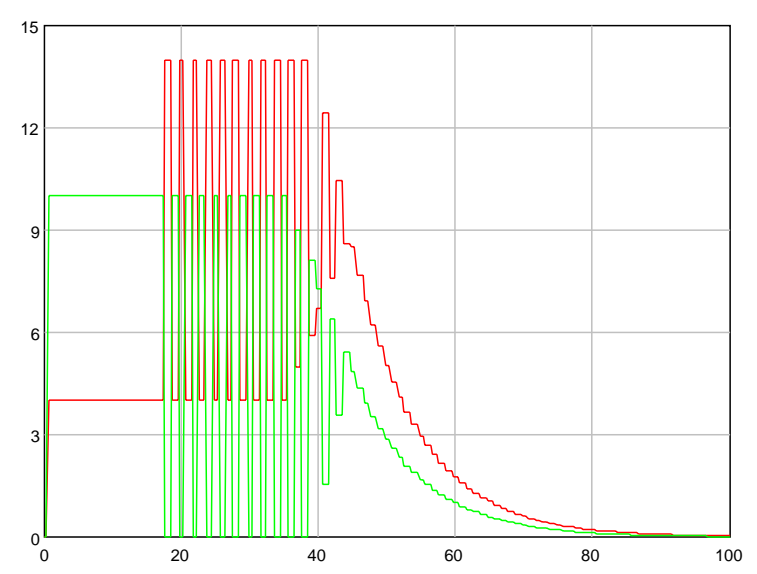

Figure 4: Production from Producer 1 (red curve) and Producer 2 (green curve) using correct strategy.

Producer 1 is initially able to satisfy all the demand. Thus, in this phase Producer 2 is completely idle. However, as the reservoir of Producer 1 is reduced, it eventually becomes necessary to activate Producer 2 as well. This happens at about $t=20$. This way it is possible to statisfy the demand for some time. However, at $t=31$ Producer 2 reaches the maximum production $b_{2}=10$, and then it becomes impossible to satisfy the demand.

In Figure 4 we have plotted the production from the two producers using the correct strategy. Again the red curve represents Producer 1 while the green curve represents Producer 2. According to the correct strategy, one should start out by giving priority to Producer 2, and let Producer 1 cover the remaining demand. As a result the state vector $\boldsymbol{R}$ approaches the straight line from the origin and through $C$. As soon as the stat vector hits this line, the strategy fluctuates between giving priority to Producer 1 and Producer 2 . This way the state eventually approaches the point $C$, which results in an optimal performance.

Note that the fluctuations in the production is a result of alternating between two extreme priorities. When the state is close to the straight line through $C$, one would normally use a more balanced approach and thus avoid these fluctuation. Still from a consumer's point of view, the result is the best one could hope for.

\section{INFLOW AND DEMAND MODELS}

In this section we introduce some possible models for representing the inflow of energy to the producers and the changing demand from the consumers. In a real-life application these models should of course be fitted using available data. Statistical methods, however, are not within the scope of the present paper. Instead we focus on models which capture the essential aspects of energy systems. All models are expressed as discrete time models, where the discrete time unit is a day.

\subsection{Inflow models}

We introduce two inflow models, one for rain and one for wind. The rain inflow model takes into account that there will be many days without any rain at all. Moreover, for a given rainy day, the amount of rain varies a lot. In order to capture these aspects we use a compound Markov chain. That is, we introduce a time-homogenous Markov chain $\left\{X_{m}\right\}_{m>0}$, with state space $\{0,1\}$, and where $X_{m}=1$ if it rains on the $m$ th day, and zero otherwise, $m=1,2, \ldots$. The transition matrix of the chain is:

$$
P=\left[\begin{array}{ll}
p_{00} & p_{01} \\
p_{10} & p_{11}
\end{array}\right],
$$

where $p_{i j}=P\left(X_{m+1}=j \mid X_{m}=i\right) \in(0,1), i, j=0,1$. The steady state probabilities of this very simple chain, denoted $\pi_{0}$ and $\pi_{1}$ respectively, are easily found as:

$$
\begin{aligned}
& \pi_{0}=P\left(X_{m}=0\right)=\frac{p_{10}}{p_{01}+p_{10}}, \\
& \pi_{1}=P\left(X_{m}=1\right)=\frac{p_{01}}{p_{01}+p_{10}} .
\end{aligned}
$$

In order to model the amount of rain we introduce the process $\left\{Y_{m}\right\}_{m>0}$, where $Y_{m}$ denotes the potential amount of rain on the $m$ th day, given that $X_{m}=1, m=1,2, \ldots$. The actual amount of rain on the $m$ th day can then be expressed as the product $X_{m} \cdot Y_{m}, m=1,2, \ldots$.

The $\left\{Y_{m}\right\}_{m>0}$-process is modelled as a transformation of a Gaussian process. That is, for suitable non-negative and non-decreasing functions $\phi_{1}, \phi_{2}, \ldots$, we may write $Y_{m}=\phi_{m}\left(V_{m}\right), m=1,2, \ldots$, where $\left\{V_{m}\right\}_{m>0}$ is a sequence of standard normally distributed variables such that $\left.\operatorname{Cov}\left(V_{m+1}, V_{m}\right)\right)=c, m=1,2, \ldots$. Such a Gaussian process is easily constructed as follows: Let $U_{1}, U_{2}, \ldots$ be a sequence of independent standard normally distributed variables. We then define:

$$
\begin{aligned}
V_{1} & =U_{1}, \\
V_{m+1} & =c V_{m}+\sqrt{1-c^{2}} U_{m+1}, \quad m=1,2, \ldots .
\end{aligned}
$$

More specifically, in cases where there is no seasonal variations in the potential amount of rain, we may e.g., choose:

$$
\phi_{m}\left(V_{m}\right)=\phi\left(V_{m}\right)=\exp \left(\mu+\sigma V_{m}\right), \quad m=1,2, \ldots .
$$

As result $Y_{m}$ becomes lognormally distributed with:

$$
\mathrm{E}\left[Y_{m}\right]=\exp \left(\mu+\sigma^{2} / 2\right)
$$

$$
\mathrm{SD}\left[Y_{m}\right]=\exp \left(\mu+\sigma^{2} / 2\right) \sqrt{\exp \left(\sigma^{2}\right)-1},
$$

for $m=1,2, \ldots$.

The wind inflow model can usually be expressed in a simpler fashion as it rarely happens that there is no wind at all on a given day. Thus, for the wind inflow model we simply use another transformation of a Gaussian process.

\subsection{The demand model}

In most locations a demand model needs to incorporate some degree of seasonal effects. An easy way to do this is to use a multiplicative model where the demand on the $m$ th day, denoted $D_{m}$ is expressed as a product $D_{m}=S_{m} \cdot Z_{m}$, $m=1,2, \ldots$. Here $\left\{S_{m}\right\}_{m>0}$ is a deterministic periodic process, while $\left\{Z_{m}\right\}_{m>0}$ is another transformation of a Gaussian process. 


\section{NUMERICAL EXAMPLES}

In this section we will illustrate the proposed approach by some numerical examples. We start out by extending the example considered in Section 3. That is, we consider the example with the two producers, Producer 1 and 2 where the parameters of the PPR-functions are $a=0.1, b_{1}=14$ and $b_{2}=10$. We also use the same initial reservoir states, i.e., $R_{1}(0)=400$ and $R_{2}(0)=350$.

As in Section 3 we start out by considering a case with a constant demand of $K=14$. In this case, however, we include inflow for the two producers using the models described in Subsection 4.1. We assume that both producers have water reservoirs, and that the rain inflow model will be used in both cases.

We let the transition matrix for the Markov chain associated with both producers be:

$$
P=\left[\begin{array}{ll}
0.9 & 0.1 \\
0.5 & 0.5
\end{array}\right]
$$

Thus, the steady state probabilities for both Markov chains are:

$$
\begin{aligned}
& \pi_{0}=P\left(X_{m}=0\right)=\frac{p_{10}}{p_{01}+p_{10}}=\frac{0.5}{0.1+0.5}=\frac{5}{6} \\
& \pi_{1}=P\left(X_{m}=1\right)=\frac{p_{01}}{p_{01}+p_{10}}=\frac{0.1}{0.1+0.5}=\frac{1}{6} .
\end{aligned}
$$

Thus, the reservoirs are on average exposed to rain one out of six days.

The covariance parameter $c$, representing the correlation between the Gaussian variables, is chosen to be 0.7 . Note that this implies that the logarithmic correlation between the amount of rain in two consecutive days with rain is 0.7 . In cases where there are several days without rain between two days with rain, the logarithmic correlation between the amount of rain is much lower.

Concerning the transformed Gaussian processes, we let $\left\{V_{m}^{r}\right\}_{m>0}$ denote the gaussian process associated with the $r$ th producer, $r=1,2$. Moreover, we let $\phi^{r}$ denote the transformation for the $r$ th producer, and assume that:

$$
\phi^{r}\left(V_{m}^{r}\right)=\exp \left(\mu^{r}+\sigma^{r} V_{m}^{r}\right), \quad m=1,2, \ldots,
$$

where $\mu^{1}, \mu^{2}, \sigma^{1}$ and $\sigma^{2}$ are chosen such that:

$$
\begin{array}{lll}
\mathrm{E}\left[Y_{m}^{1}\right]=45.0 & \text { and } & \mathrm{SD}\left[Y_{m}^{1}\right]=9.0 \\
\mathrm{E}\left[Y_{m}^{2}\right]=30.0 & \text { and } & \mathrm{SD}\left[Y_{m}^{2}\right]=6.0 .
\end{array}
$$

In a situation with inflow, we also need to include restrictions on the maximum amount of energy that can be stored in a reservoir. Whenever the amount of energy stored in a reservoir reaches this level, the surplus energy needs to be removed. Typically this implies that the water is let out into a river without running through the power plant. During the simulations we will keep track of this energy loss. We let $M^{r}$ denote the maximum amount of energy that can be stored in the reservoir of the $r$ th producer, $r=1,2$, and assume that $M^{1}=600$ while $M^{2}=500$.
While the production strategies considered in Section 3 were intended to be used in systems with zero inflow, we will still use the same strategies in the case with positive inflow as well and compare the results.

We are now ready to run a simulation using the specified models. Since the inflow models include uncertainty we need to run a Monte Carlo simulation where the same model is simulated many times. More specifically, we run $N=10000$ simulation of the system. In each simulation we monitor the system over a period of 3600 days.

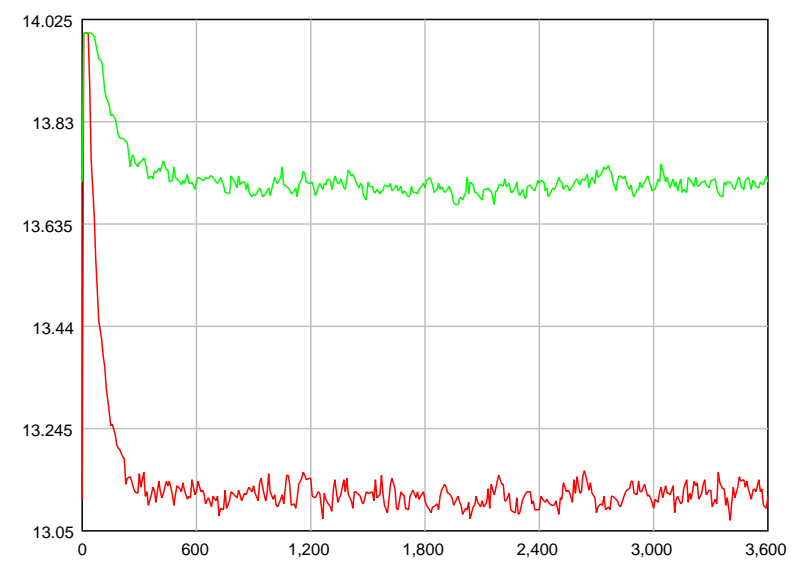

Figure 5: Average total production using correct strategy (green curve) and incorrect strategy (red curve).

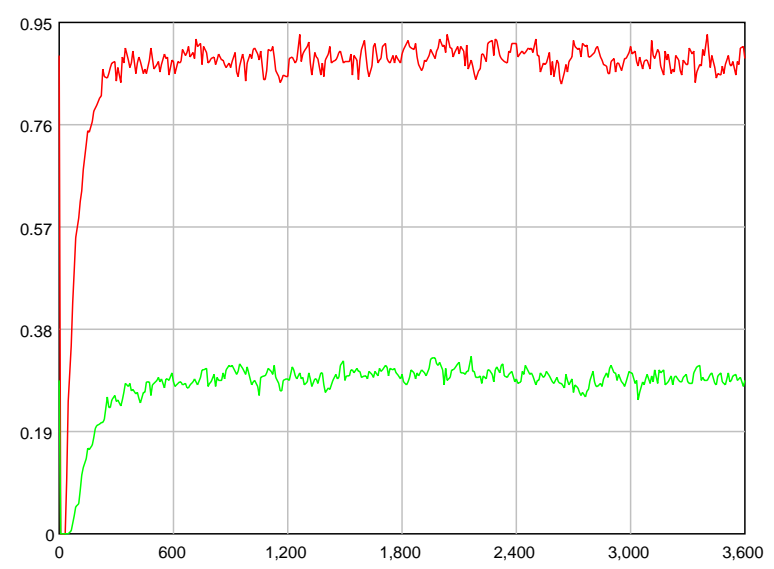

Figure 6: Average missing energy production using correct strategy (green curve) and incorrect strategy (red curve).

The resulting average production using the two strategies are shown in Figure 5. We observe that using the correct strategy the average production appears to stabilize around 13.7 per unit time which is about $98 \%$ of the total demand. On the other hand if the incorrect strategy is used, the average production appears to stabilize around 13.1 per unit time which is about $94 \%$ of the total demand. In Figure 6 we have plotted the average missing energy production for the two strategies. We see that the curve for the incorrect strategy is significantly higher than the curve for the correct strategy. Thus, we conclude that the best strategy for the case with no inflow appears to perform best in the case with inflow as well. 


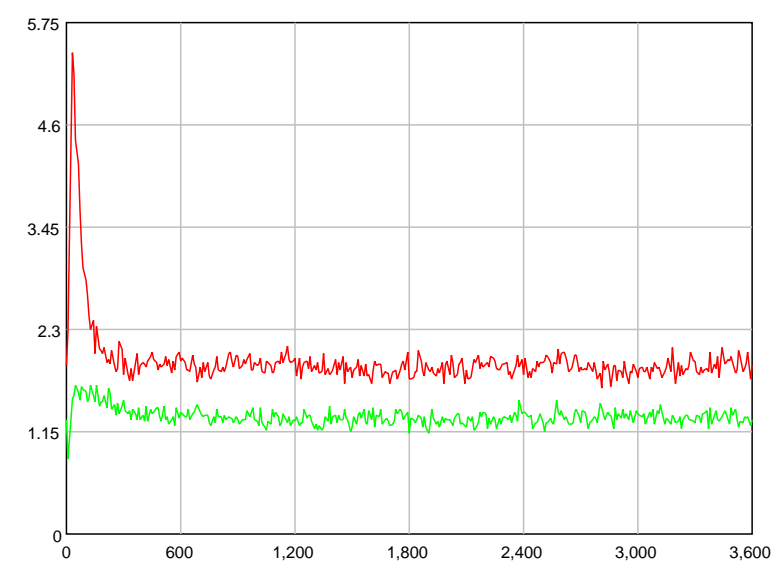

Figure 7: Average total energy loss using correct strategy (green curve) and incorrect strategy (red curve).

In Figure 7 we have plotted the average total energy loss as a result of surpluses in the reservoirs. Again the correct strategy performs better with an average loss of about 1.3 per unit time compared to the incorrect strategy with an average loss of 1.9 per unit time.

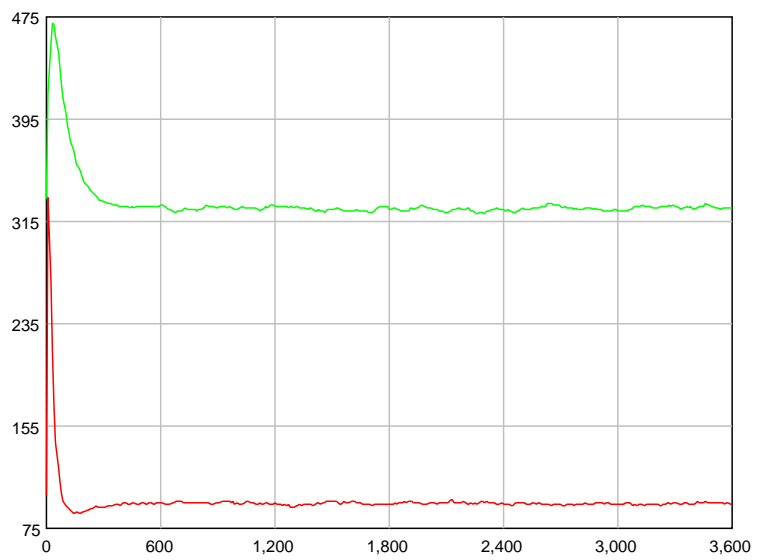

Figure 8: Average reservoir levels for Producer 1 (red curve) and Producer 2 (green curve) using incorrect strategy.

The difference between the two strategies is also very evident when we compare the average reservoir levels for the two producers. See Figure 8 and Figure 9. We see that by using the incorrect strategy, the average reservoir levels for Producer 1 and 2 are respectively 94 and 325. The corresponding average levels using the correct strategy are respectively 370 and 260 . We recall that the incorrect strategy gave priority to Producer 1 . As result the average reservoir level for Producer 1 is much lower compared to the level using the correct strategy. We also observe that the correct strategy tends to obtain a much better balance between the reservoir levels for the two producers.

We now extend this example further by including a stochastic demand. This is done by using the multiplicative model described in Subsection 4.2. More specifically we let process $\left\{S_{m}\right\}_{m>0}$ follow a sine curve with period 360 days, mean value equal to the constant $K=14$, and

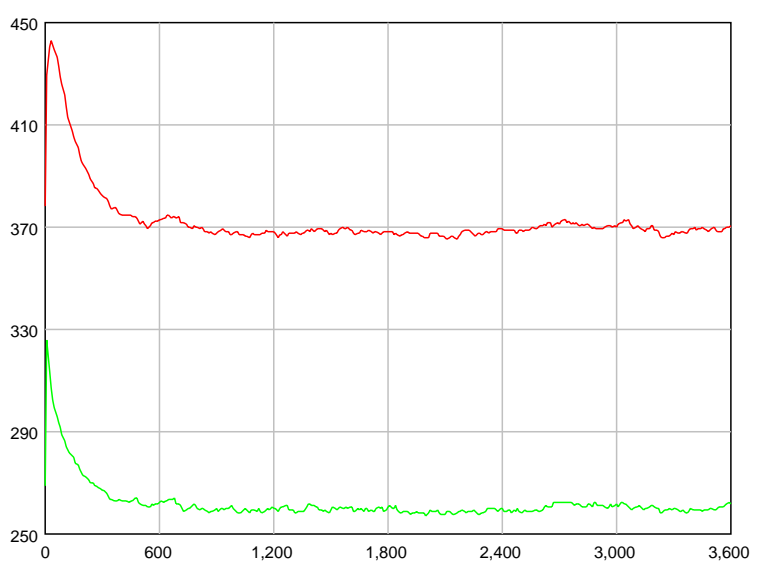

Figure 9: Average reservoir levels for Producer 1 (red curve) and Producer 2 (green curve) using correct strategy.

amplitude 6 . Moreover, for the process $\left\{Z_{m}\right\}_{m>0}$ we use a transformation of a Gaussian process with correlation 0.7. Again we use an exponential transformation so that the resulting process is a sequence of lognormally distributed variables with mean value 1 and standard deviation 0.2 .

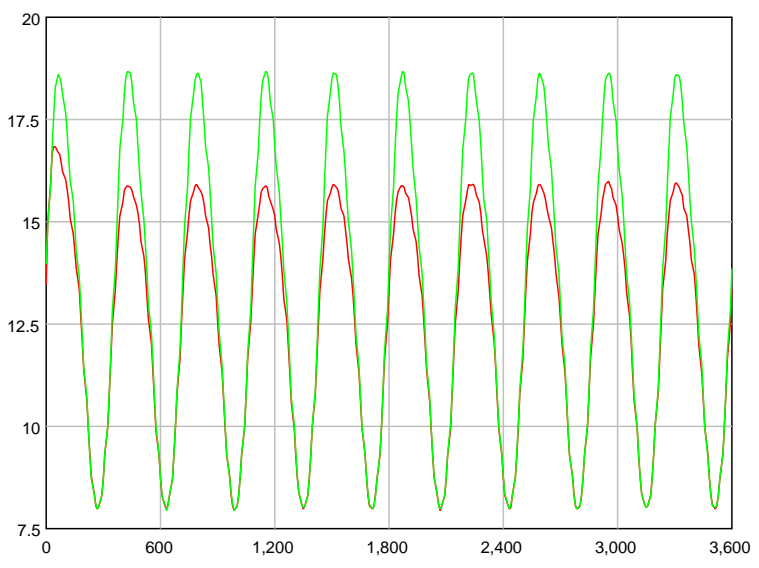

Figure 10: Average total production using correct strategy (green curve) and incorrect strategy (red curve).

The results are shown in Figure 10 and Figure 11. We see that the correct strategy performs significantly better than the incorrect strategy in this case as well. The average total production is higher using the correct strategy compared to the incorrect strategy, at least in periods where the demand is high. Similarly the average missing energy production is lower using the correct strategy compared to the incorrect strategy.

Finally, we include an example where we add a third producer based on wind power. For this producer the inflow is modelled using the wind inflow model applying an exponential transformation of the Gaussian process. Thus, we again get a sequence of lognormally distributed variables. For this producer the mean value is 4.0 and the standard deviation is 1.0. Since wind energy cannot easily be stored, we assume that there is no reservoir for this producer. Due to the lack of a reservoir, we assume that this producer is given priority before the other producers. In or- 


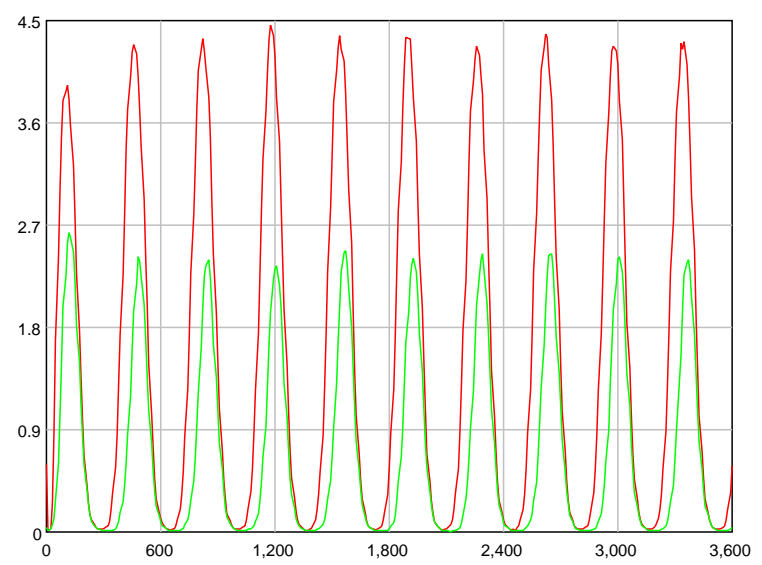

Figure 11: Average missing energy production using correct strategy (green curve) and incorrect strategy (red curve).

der to prioritize between the producers with reservoirs, the strategies from the previous examples are used once again.

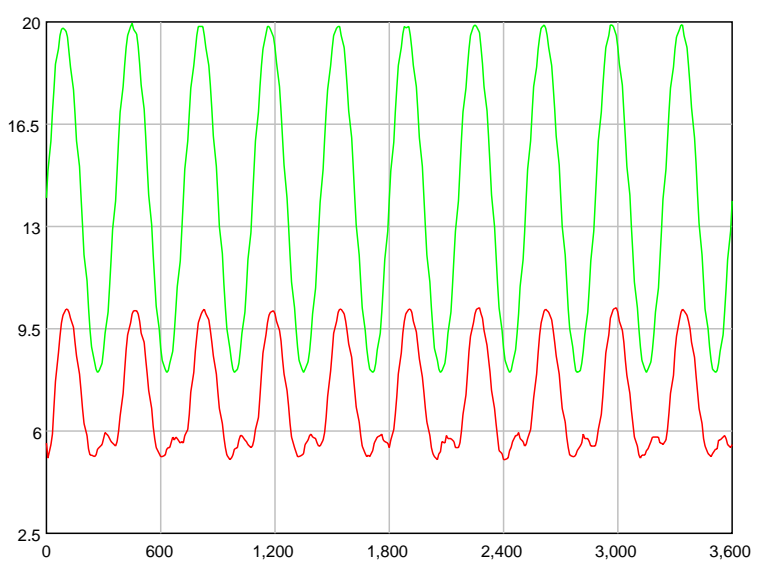

Figure 12: Average total production using correct strategy (green curve) and incorrect strategy (red curve).

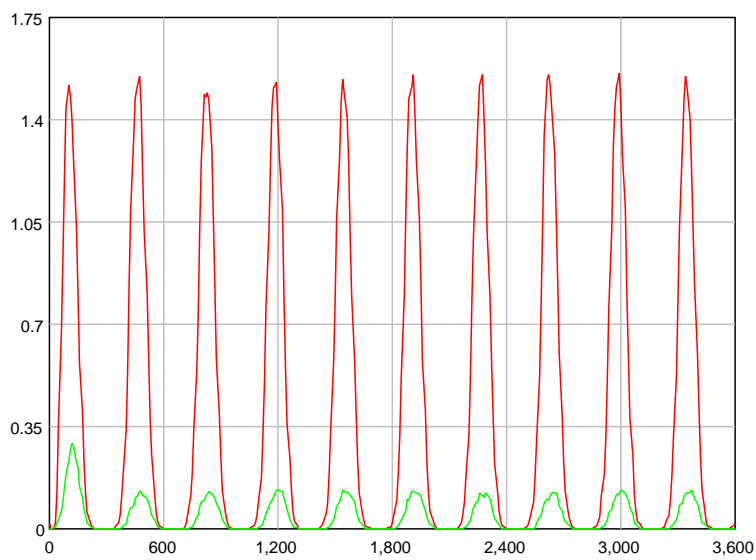

Figure 13: Average missing energy production using correct strategy (green curve) and incorrect strategy (red curve).

The results are shown in Figure 12 and Figure 13. With a third producer available, the average available production capacity is almost equal to the average demand. When the correct production strategy is used, the average missing energy production is almost zero. Thus, we conclude that the correct strategy performs much better than the incorrect strategy in this case as well.

\section{CONCLUSIONS}

In the present paper we have introduced various models for producers and consumers in an energy system. By considering a simplified problem with zero energy inflow and constant demand we have identified a reasonable production strategy. For the simplified case this strategy is in fact optimal. In the more realistic case with energy inflow and stochastic demand, the strategy still performs very well. The same holds true if we introduce a third producer with no reservoir. Finding an exact optimal production strategy in the general case is a very difficult problem. Thus, having a reasonable candidate is always useful.

All the calculations are carried out using software implemented in java where all the producers and consumers are implemented as independent objects interacting with each other. This methodology is very well suited for analysing the complexity of large scale energy systems.

Future work in this area includes a closer investigation of the performance of the identified strategy in more general settings, as well as models where energy prices and power grid infrastructure is taken into account.

\section{REFERENCES}

Axelrod, R. The complexity of cooperation: agentbased models of competition and collaboration. Princeton University Press, 1997.

Huseby, A. B. and Haavardsson, N. F. Multi-reservoir production optimization. European Journal of Operational Research, (199), 236-251, 2009.

Huseby, A. B. and Haavardsson, N. F. Multi-reservoir production optimization under uncertainty. In $R e$ liability, Risk and Safety. Theory and Applications, CRC Press, 407-413, 2010.

Kremers, E. Modelling and Simulation of Electrical Energy Systems through a Complex Systems Approach using Agent-Based Models. KIT Scientific Publishing, 2013.

Palensky, P. and Dietrich, D. Demand Side Management: Demand Response, Intelligent Energy Systems, and Smart Loads. IEEE Transactions on Industrial Informatics, 7(3): 381-388, 2011. 